\title{
Ablehnung oder Zuwendung? - Zum Umgang der Psychoanalyse mit Homosexualität
}

\author{
Victoria Preis (Berlin)
}

\begin{abstract}
Zusammenfassung: Der Umgang der Psychoanalyse mit Homosexualität ist von Ablehnung und Pathologisierung geprägt. Obwohl aktuell vermehrt Auseinandersetzungen auf Tagungen und Veranstaltungen sowie in Zeitschriften stattfinden, in denen die lang anhaltende Homophobie seitens der Psychoanalyse kritisiert sowie eine Beschäftigung mit dem «homophoben Erbe» gefordert wird und sich damit eine mögliche Offenheit abzeichnet, sind eine skeptische bis ablehnende Haltung seitens vieler Psychoanalytiker_innen anhaltend an der Tagesordnung. Im Artikel werden sowohl diese ablehnende wie auch eine offene Haltung gegenüber homosexuellem Begehren in unterschiedlich grossem Umfang betrachtet. Dabei richtet sich der Fokus auf die Frage, was mit diesem Begehren assoziiert wird - aufPhantasien von Psychoanalytiker_innen über homosexuelle Sexualität-sowie darauf, welche mögliche Funktion die Figur des Homosexuellen für die Psychoanalyse haben könnte.
\end{abstract}

Schlüsselwörter: Homosexualität, Homophobie, Psychoanalyse, Abwehr, Sexuelles

\section{Einleitung}

Obwohl Homophobie und Diskriminierung im Leben von Homosexuellen anhaltend präsent sind und es derzeit massive reaktionäre Strömungen in Bezug auf die Beurteilung der Homosexualität gibt, wird seit den 1990er Jahren eine Normalisierung der homosexuellen Sexualität in sogenannten westlichen Gesellschaften festgestellt. So gebe es derzeit ein diverses "Angebot» von zur Verfügung stehenden Sexualitäten, weshalb Homosexualität «den Charakter des ganz Anderen verloren» habe (Eder, 2010, S. 169) und zum Normalfall geworden sei. ${ }^{1}$ Weiter habe sich ein Wandel der rechtlichen Landschaft in Bezug auf die Bewertung von Partnerschaften und Sexualitäten vollzogen, so z. B. die Liberalisierung der gleichgeschlechtlichen Ehe oder eine Ausweitung der Adoptionsmöglichkeiten für gleichgeschlechtliche Paare in einigen Ländern (Pechriggl, 2012, S. 83). In diesem Zusammenhang wird nicht nur ein Bedeutungswandel der Homosexualität, 
sondern von Sexualität ${ }^{2}$ im Allgemeinen festgestellt. So bemerkt Gunter Schmidt ein Verschwinden der Sexualmoral, an deren Stelle eine Konsensoder Verhandlungsmoral getreten sei (1996, S. 11), womit eine Rationalisierung sowie eine Regulierung der Sexualität erfolgen (ebd., S. 13). Gleichzeitig gingen Leidenschaft, Irrationalität und die «Sprengkraft» (Sigusch, 2013, S. 173) verloren - Eigenschaften, die ehemals mit der Sexualität assoziiert wurden. Volkmar Sigusch sieht eine Entwicklung hinsichtlich sexueller Praktiken, der zufolge «Aggression, Macht, Kampf, Übergriff, Risiko und Triumph über Gefahr [...] auf dem Weg [seien], anstößig zu werden» (2013, S. 222). Insa Härtel wiederum fasst gerade diese Feststellungen des Leidenschaftsschwindens und dass das «triebhafte Sexuelle» bedeutungslos geworden sei (Sigusch, 2013, S. 229, zit.n. Härtel, 2014, S. 101) als eine «kulturelle Abwehrbewegung» eben gerade gegen den Trieb und das Sexuelle (Härtel, ebd.).

Auch in der Psychoanalyse wird seit Langem eine schwindende Bedeutung des Sexuellen für die Theorie und Praxis festgestellt (Green, 1995, Heenen-Wolff, 2009), und mitunter ebenso als Abwehr gegen das Sexuelle verstanden (Zamanian, 2011). ${ }^{3}$ Mit der Abkehr von der Sexualität in der Psychoanalyse wurde auch die Überlegung verbunden, die Bewertung der Homosexualität als pathologisch würde abnehmen (Heenen-Wolff, 2009, S. 173). Tatsächlich wird, bezogen auf die letzten 15 Jahre, ein Wandel im Umgang mit der gleichgeschlechtlichen Orientierung und Lebensweise festgestellt (Rauchfleisch, 2014, S. 381). Zudem finden aktuell Fragen um (Homo-)Sexualitäten auf Tagungen, Veranstaltungen sowie in Zeitschriften Eingang (u.a. PSYCHE, 2015), auf und in welchen eine Beschäftigung mit dem «homophoben Erbe» gefordert wird (Quindeau, 2015, S. 648). Dennoch gibt es nach wie vor Hinweise auf eine anhaltende Diskriminierung von und auf eine teils ablehnende Haltung gegenüber homosexueller Sexualität: So etwa ein im Januar 2015 erschienener Bericht in der tageszeitung über einen Psychoanalytiker, der wegen dem Versuch, eine sogenannte Konversionstherapie durchzuführen, von einem ehemaligen Patienten verklagt wird (Keller, 2015), oder der Schulterschluss einiger Psychoanalytiker_innen mit den Gegner_innen der sogenannten HomoEhe oder Ehe für alle in Frankreich (Pechriggl, 2012; v. Braun, 2014).

Autor_innen, die versucht haben, Homophobie in der Psychoanalyse zu verstehen, haben die Hypothese entwickelt, dass es sich dabei um die Abwehr einer wahrgenommenen Bedrohung oder Konfrontation handelt. So wurde eine empfundene Bedrohung der Identität männlicher Psychoanalytiker vermutet sowie die potenzielle Konfrontation mit der eigenen latenten Homosexualität durch die manifeste Homosexualität (Dannecker, 2014, S. 78). ${ }^{4}$ Ebenfalls von einer Abwehr 
seitens Psychoanalytiker_innen ausgehend, will ich an dieser Stelle weniger auf die Aspekte der Identität fokussieren. Vielmehr will ich den derzeitigen psychoanalytischen Diskurs um homosexuelles Begehren auf eine von Härtel beschriebene Abwehrbewegung gegen das Sexuelle untersuchen. Gibt es demnach einen Zusammenhang zwischen dem Stand des Sexuellen in der Psychoanalyse und der aktuell anhaltend erregten psychoanalytischen Diskussion um Homosexualität? Dafür versuche ich zu begreifen, an welchen Stellen homosexuelles Begehren (unbewusst) genau mit diesem Abgewehrtem konfrontieren könnte, um daran anschliessend einer potenziellen Funktion der Homophobie für die Psychoanalyse nachzugehen.

Im weiteren, kürzeren Teil werde ich die aktuell zuteilen offenere Auseinandersetzung mit Homosexualität sowie die Kritik an der Homophobie einer Betrachtung unterziehen und Fragen nach möglichen Ursachen für die aktuelle Beschäftigung in der Psychoanalyse mit unter anderem «sexuellen Verhältnissen» und der Kritik an der Homophobie stellen.

\section{Zur aktuellen (homophoben) Diskussion}

Offen ablehnende Haltungen gegenüber homosexuellem Begehren sind derzeit in der Psychoanalyse wenig zu hören und erweisen sich folglich als schwer erkennbar, da Vorurteile gegenüber Homosexuellen wegen der Political Correctness häufig kaum bis gar nicht mehr öffentlich geäussert werden (Heenen-Wolff, 2009, S. 174). Dass diese nach wie vor bestehen, zeigt sich vielmehr darin, dass Schwule und Lesben weiterhin damit rechnen müssen, nicht zur psychoanalytischen Ausbildung zugelassen zu werden (Rauchfleisch, 2014; Pechriggl, 2012), weshalb sie in den psychoanalytischen Institutionen wenig präsent sind (Quindeau, 2015, S. 655). Überdies müssen Homosexuelle, sofern sie in einer Paarbeziehung leben, stets nachweisen, dass diese «dem Ideal einer sich gegenseitig achtenden Liebe entspricht» (Dannecker, 2014, S. 77).

Als beispielhaft für diesen derzeitigen Umgang erweist sich die Debatte um die Homo-Ehe sowie die Möglichkeiten der Adoption und der künstlichen Befruchtung für gleichgeschlechtliche Paare. In diesen Diskussionen wird sich zwar kaum offen homophob geäussert, und es wird sogar der Anspruch erhoben, sich deutlich von solchen Positionen abzugrenzen (Vacquin \& Winter, 2013, S. 86). ${ }^{5}$ Auch den im Folgenden wiedergegebenen Positionen zweier französischer Psychoanalytiker_innen und eines deutschen Psychoanalytikers, die zu Homosexualität, homosexueller Ehe und Elternschaft sowie zu künstlicher Befruchtung Stellung beziehen, ist gemein, dass diese nicht zwangsläufig als offen 
homophobe Äusserungen deklariert werden können. Gleichwohl sind die dargelegten Gründe und dahinterliegenden Motive, die gegen die Legalisierung der HomoEhe angeführt werden, vergleichbar mit ehemals offen geäusserten homophoben Positionen und verweisen auf eine Skepsis oder ablehnende Haltung gegenüber homosexuellem Begehren. So wird in der Forderung der Ehe für alle der Angriff auf Institutionen und Grenzen gesehen und entsprechend der Niedergang der Gesellschaft und der Zivilisation befürchtet. Dieser Angriffgehe von einer erpresserischen, steuernden, «winzige[n] Minderheit» aus (Vacquin \&Winter, 2013, S. 84). Als diese Minderheit werden nicht nur Homosexuelle beschrieben, die das Recht auf Ehe fordern, sondern auch diejenigen, die Monette Vacquin und Jean-Pierre Winter als politisch korrekte Besessene bezeichnen. Homosexuelle, die die Ehe für alle wünschen, betrachten sie dabei hauptsächlich als Symptom der Krise (ebd.). Die Autor_innen können den Wunsch nach einer Schaffung von Rechtsformen für homosexuelle Partnerschaften im Sinne von Partnerschaftsverträgen nachvollziehen. Dass das Recht auf Ehe oder künstliche Befruchtung gefordert wird, gilt ihnen jedoch als Weigerung, «Leben, Institutionen und Werte [...]» (ebd., S. 84) weiter zu geben und sei eine «antizivilisatorische Attacke auf unsere Institutionen» (ebd., S. 87). Denn die Ehe für alle sei als ein Wunsch zu verstehen, «Vater und Mutter» zerstören zu wollen (ebd., S. 84). Die Autor_innen sehen damit das Konzept von heterosexueller Ehe und Familie durch Homosexuelle bedroht. Auf eine empfundene Bedrohung der Bedeutung der männlichen Identität im Sinne des Vaters, und dessen Rolle in den neuen Familienformen könnte auch die Sorge verweisen, die Bedeutung des Vaters und der Vaterschaft generell würden in neuen Familienformen anonymisiert und marginalisiert (Metzger, 2015a, S. 4 f.). Das vermutete Verschwinden des Vaters, dem die Rolle des Begrenzenden zugeordnet wird, wird zudem als ein Verlust von Bezugsrahmen verstanden. Darüber hinaus wird seitens der Kritiker_innen der Ehe für alle dieVermutung aufgestellt, homosexuelle Paare würden bei der künstlichen Befruchtung das gegengeschlechtliche Elternteil ablehnen, was als Ausschluss eines Dritten zu bewerten sei (ebd., 2015a, S. 4). ${ }^{6}$

Es besteht weiter die Sorge, die Familie würde zu einer künstlichen Angelegenheit. Denn dass homosexuelle Paare mittels künstlicher Befruchtung generativ werden, wird als «medizinische Manipulation» diskutiert (ebd., S. 5). Weiter wird homosexueller Elternschaft eine egoistische Motivation unterstellt. In Kontrastierung zur Kleinfamilie mit einer liebenden, zu Bindung fähigen Mutter und einem begrenzenden Vater wird homosexuellen Eltern vorgeworfen, das Wohl der Kinder und deren «reale Bedürfnisse» zu missachten und für ihre selbstbezo- 
genen Wünsche die Verwissenschaftlichung menschlicher Bindungen in Kauf zu nehmen (Vacquin \& Winter, 2013, S. 88).

Die Ermöglichung der künstlichen Befruchtung für homosexuelle Paare sei weiter als der Versuch zu verstehen, Grenzen z. B. der Geschlechter und damit die der Natur zu zerstören (ebd., S. 87 f.).ZZudem werden den Befürworter_innen der Homo-Ehe, den «neuen Sexualitäten» (Metzger, 2015b, S. 4) und pauschal «den Gendertheorien» (ebd., S. 5) Grössen- und Allmachtphantasien unterstellt. Bisexualität im Sinne der Geschlechtsidentität wird hier beispielsweise als die Unfähigkeit verstanden, die Begrenztheit des eigenen Geschlechts zu akzeptieren und einzusehen, «dass nicht alles möglich sein wird» (ebd., S. 3).

Die Feststellung des vermeintlichen Ausschlusses eines gegengeschlechtlichen Dritten in der homosexuellen Beziehung wirft die Frage auf, weshalb man sich ausschliesslich um den Ausschluss eines heterosexuellen Gegenübers sorgt. Von einem bewussten Ausschluss zu sprechen, stärkt Hans-Geert Metzgers Annahme einer vermeintlich heterofeindlichen Stimmung und vermittelt den Eindruck, homosexuelles Begehren und Elternschaft fussten lediglich auf Ausschlussbedürfnissen gegenüber der Heterosexualität. Dass dieses Ausschlussbedürfnis nur homosexuellen Paaren unterstellt wird, zeigt sowohl wie sehr Metzger von einer heterosexuellen Norm ausgeht und alles jenseits dieser Norm anzweifelt, als auch wie wenig diese Norm in ihrer gesellschaftlichen Verfasstheit hinterfragt wird.

Eine ablehnende Haltung gegenüber homosexuellem Begehren zeigt sich weiter darin, dass die künstliche Befruchtung bei Heterosexuellen kaum Aufmerksamkeit erlangt, sie allerdings «bei den Homosexuellen zum Skandal und zum Generalangriff auf die Natur» gemacht wird, wie Tjark Kunstreich kritisch bemerkt (2015, S. 43).

Den Gendertheorien, und sinnbildlich dafür Judith Butler, pauschal die Ablehnung der ödipalen Ordnung sowie eine «Heterofeindschaft» zu unterstellen (Metzger, 2015b, S. 4), vermittelt den Eindruck eines empfundenen Angriffs auf diese Ordnungen durch Homo- und Bisexualität. Die Einschätzungen der Bisexualität als einer narzisstischen Allmachtphantasie, der Homo-Ehe und -Elternschaft als Ausschluss eines Dritten und Angriff auf Institutionen und Grenzen, verweisen demnach auf die Vorurteile und Ablehnung gegenüber homosexuellem oder bisexuellem Begehren.

Warum wird der Wunsch nach homosexueller Ehe und Elternschaft als Angriff und Bedrohung wahrgenommen, und warum kommt es zu einer aufreibenden Diskussion um Grenzenlosigkeit, Heterofeindschaft und um die vermeintliche Steuerung einer Minderheit? Was wäre weiter das Interesse oder vielmehr 
der Gewinn, ein solches Bild des Grenzenlosen zu zeichnen und sich gleichzeitig davon abzugrenzen?

\section{Zur Abkehr vom Sexuellen}

Robert Pfaller stimmt in weiten Teilen den Positionen zu, dass sich mit einer Entkoppelung von Sexualität und Fortpflanzung die Differenz der Geschlechter und der Generationen sowie die Bedeutung von «strikten «väterlichen` Zwängen» verändern würden (2005, S. 9 f.). Ebenso bemerkt er eine «Ablösung der Sexualpraktiken von den Institutionen» (ebd., S. 11). Im Vergleich zu den soeben untersuchten Autor_innen versteht Pfaller «Vater» allerdings strukturell, und der veränderte Umgang mit Institutionen wird von ihm weder als Bedrohung inszeniert noch wird eine Gruppe dafür verantwortlich gemacht. Vielmehr sieht er diese Entwicklung als Ausdruck einer derzeitigen als narzisstisch zu bewertenden gesellschaftlichen Tendenz, die dem Imperativ folge, in jeder Situation authentisch zu sein. Alles von aussen an das Individuum Herantretende werde auf die "Konformität mit dem Ego» geprüft (ebd., 2009, S. 34) und sich anhaltend gefragt: Was bedeutet das für mich? Alles, was nicht mit dem Ich in Übereinstimmung gebracht werden könne, werde als entfremdend oder sogar gewaltvoll wahrgenommen und «[d] er narzisstischen Tendenz entsprechend wird jegliche Alterität verpönt», weshalb nur das, «womit die volle Identifizierung möglich ist» (ebd., 2005, S. 12), erlaubt sei. Pfaller spricht auch von einer «narzisstischen «Intimisierung» (ebd., S. 10).

Diese Entwicklung wirke sich auch auf die Beurteilung der Sexualität aus. Denn, psychoanalytisch gesehen, sei das Begehren immer auch das Begehren des Anderen und stehe daher zwangsläufig für Alterität (ebd., S. 12). Laplanche versteht das Sexuelle als das Resultat einer vollzogenen Verführung (1996), weshalb sich nach Härtel im Sexuellen auch das Andere, quasi als Fremdkörper, zeige (Härtel, 2014, S. 102). Als etwas Fremdes und Fremdbestimmendes und nicht Ichkonform Wahrgenommenes werde Sexualität, so Pfaller, als «Entfremdung erlebte 〈Alterität» verstanden (2005, S. 10). Weil aber das Fremde und entsprechend jegliche Alterität nicht mit dem Bedürfnis nach Subjektivität und Autonomie vereinbar seien und entsprechend geächtet würden, werde versucht, «die als belästigend empfundene Sexualität [...] zu verbannen» (ebd., 2009, S. 36). Die «Fremdheit im sexuellen Wollen» sei derzeit wenig akzeptabel (Härtel, 2014, S. $101 \mathrm{f}$.).

Der Sexualität gegenüber stünde das Ideal der Asexualität, die als natürlich und authentisch empfunden werde. Pfaller sieht diese auch als das Resultat eines Aushandlungskampfes. Denn die Fähigkeit zu begehren sei eine Beute, die nicht allen zur Verfügung stehe. Die Verlierer dieses Kampfes «beginnen [...] von sich 
aus die Beute abzulehnen oder den Verzicht libidinös zu besetzen». Damit sei die Asexualität eine Form der «Libidounterbringung» (ebd., S. 40) und demnach die Antwort auf oder Lösung eines Verteilungskampfes.

Sofern das Sexuelle folglich abgewehrt wird, wäre die Frage, an welchen Stellen das Sexuelle untergebracht sein könnte oder wer derzeit die Möglichkeit hat, zu begehren?

Leo Bersani konstatiert, dass mit dem passiven homosexuellen Sex (beim Mann) Weiblichkeitund Krankheitbzw. Tod assoziiert würden; der schwule passive Mann übernehme (beim Sex) - im Rahmen dieser Phantasie - widerstandslos die Rolle der Frau (1988, S. 54 f.). Penetriert werden bedeutet Bersani zufolge in diesem Zusammenhang, sich der Macht zu entsagen, was als eine «radikale Desintegration bzw. Erniedrigung des Selbst» erfahren werde (Härtel, 2014, S. 104). Wenn dieser mit Frauen assoziierte Akt von Männern durchgeführt werde, wirke dies «(kulturell) bedrohlich» (ebd.). Dennoch übe das Gefühl, ausgeliefert und desintegriert zu sein, eine Faszination und den Wunsch nach Wiederholung aus;

insofern also die Erfahrung zuallererst zerrüttender sexueller Erregung wiederholt werden soll, begründet sich quasi die Erfahrung des Subjekts von sich - als Subjekt des «zunichte machenden» Genießens. (Härtel, 2014, S. 103)

In diesem Wunsch nach Desintegration und dem damit verbundenen Risiko verortet Härtel das Sexuelle und versteht mit Bezug auf Bersani die Figur des männlichen Homosexuellen als eine Figur, in der das abgewehrte Sexuelle aufscheine. «Männliche Homosexualität preise in diesem Sinne das Risiko des Sexuellen - als Risiko einer «Abberufung〉 des Selbst» (ebd., S.105) und bringe damit das Sexuelle an einen Platz, wo es sich als eine Figur manifestiere, in welcher sich «kulturell Abgewehrtes» zeige und «in der die Anfälligkeit für die «Gefahren», die sich mit der Sexualität kulturell zu verbinden scheinen, aufscheinen kann» (ebd., S. 110). ${ }^{7}$ Darüber hinaus sei der Schwule, der sich penetrieren lässt, nach Bersani das avantgardistische Subjekt, weil er die weibliche, mit dem Tod assoziierte Position einnimmt und damit in der Position sei, sich feminisieren zu lassen, ohne weiblich zu sein, weshalb der «Wunsch nach Selbst-Auflösung [...] Symptom eines Privilegs» sein könne (Härtel, 2014, S.108). 


\section{Reizvolle Bedrohung}

Inwiefern lässt sich in der Psychoanalyse generell, und in der Diskussion um die Ehe für alle eine solche Abwehr des Sexuellen feststellen?

Mit Verweis auf Freud, der sowohl die psychoanalytische Metatheorie als auch die «Hysterika» mit einer Hexe verglich (Freud, 1937c, S. 69), der das Sexuelle, «das überschießende, unpassende, unverständliche, anstößige Sexuelle» gemein sei, stellt Christine Kirchhoff die Abkehr von der Metatheorie fest und vergleicht diese Abwendung mit einer «Hexenjagd» (2012, S. 362 f.). Denn mit dem Verbannen der Metatheorie würde auch die «beunruhigende Sexualität ausgetrieben» (ebd.). Kaveh Zamanian vermutet eine Abwehr und spezieller eine Verleugnung der Sexualität und der unedlen, niederträchtigen Impulse in der psychoanalytischen Theorie. Die Präsenz von Liebe und Intimität werden dem Sexuellen und den Aggressionen vorgezogen, während Letztere wiederum ausagiert würden (2011, S. 44). Die Beobachtung von Ofra Shalev und Hanoch Yerushalmi, wonach Sexualität in Psychotherapien als Abwehr gegen "deeper and more difficult issues" (2009, S. 343 ff., zit.n. Zamanian, 2011, S. 38), in der Arbeit mit Patient_innen als hinderlich, sowie das Ansprechen sexueller Themen durch Patient_innen als belästigend wahrgenommen werden, könnte auf die von Pfaller angesprochene Prüderie verweisen, wonach Sexualität als fremd, störend und übergriffig aufgefasst wird.

Die Diskussion um die Bedrohung bei Vacquin und Co. verweist ebenfalls auf eine Abwehr des Sexuellen. So fällt auf, dass der Wunsch nach der Ehe für alle als «antizivilisatorische Attacke» und Zerstörung der Institutionen wahrgenommen wird. Dabei ist jedoch fraglich, wie es zu einer solchen Wahrnehmung kommt und warum dies so sehr erregt. Aus dem Wunsch nach dem Zugang zur Ehe werden ein Zerstörungswunsch und eine Entgrenzung abgeleitet, was dem traditionellen Konzept der Ehe entgegensteht, welches im Sinne des Treueversprechens für Verbindlichkeit steht und begrenzend ist. Der vermeintliche Angriff wird zudem lediglich als von den Befürworter_innen der Homo-Ehe ausgehend gesehen. Dabei wird die eigene Aggressivität verkannt, mit der auf den Wunsch nach der Ehe für alle reagiert wird. Gleichzeitig werden Vorwürfe des Egoismus, der Selbstbezogenheit und der mangelhaften Liebe gegenüber homosexueller Elternschaft erhoben und in Kontrast zur heterosexuellen Kleinfamilie gestellt sowie in übertriebenem Masse die Bedeutsamkeit von Intimität und Liebe betont. Dies könnte Ausdruck einer narzisstischen Intimisierung sein, in der sowohl jegliche Alterität abgelehnt sowie Liebe und Intimität präferiert werden.

Der Vorwurf der Attacke und Zerstörung und der empfundene Angriff auf bestehende Strukturen und damit auf deren Stabilität könnte weiter gedacht auch 
als ein Angriffdes Sexuellen mit seiner desintegrierenden Qualität - die wiederum einen Angriff auf das Ich darstellt - auf die gesellschaftliche Ordnung und deren Berechenbarkeit verstanden werden.

Die vermutete Allmacht oder Steuerung durch eine Minderheit könnte auf eine Angst vor Passivität und der eigenen Ohnmacht verweisen, wofür wiederum das Sexuelle steht. Weil das Sexuelle im laplanchen Sinne zudem auf einer Grenzüberschreitung oder einem Übergriff fusst, nämlich der unbewussten Botschaften der Eltern an das Kind, wäre das Sexuelle in einem Grenzbereich zu verorten und als ein Angriff auf oder eine Überschreitung von Grenzen zu sehen. Genau an diesem Punkt wird die Bedrohung seitens der homophoben Autor_innen diskutiert. So wird zum einen das Verschwinden der Grenzen befürchtet. Zum anderen werden den Befürworter_innen der Ehe für alle sowie der Bisexualität generell Grössenphantasien, Allmachtwünsche und die Unfähigkeit, Grenzen zu akzeptieren, unterstellt. Es könnte sich somit auch um eine Angst vor Überschreitung des Sexuellen handeln, wenn die Differenz zu denjenigen, bei denen es verortet wird, nicht ausreichend gezogen wird. Wenn das abgewehrte Sexuelle aufscheint, könnte eine solch ausgeprägte Grenzziehung gegenüber der Homo-Ehe einen Versuch darstellen, sich von dem potenziellen Ausgeliefertsein gegenüber dem Sexuellen abzugrenzen.

Geht man weiter davon aus, dass diese bedrohten Grenzen ebenfalls gesellschaftlich gezogen und begründet sind, so würde das Überschreiten vielleicht nicht nur im Gegensatz zu gezogenen Grenzen stehen, sondern im Akt von Überschreitungen würden die Grenzen zugleich auch mitkonstituiert und potenziell verschoben - auch diese Phantasie könnte als bedrohlich erlebt werden. ${ }^{8}$ Daher stellt sich die Frage, ob sich der empfundene Angriff im Sinne eines Verteilungskampfes auch auf das potenzielle Verschieben der Grenzen bezieht. In der Darstellung der Anderen als Grössenwahnsinnige und sich allmächtig Fühlende könnte eine Sorge vor deren Zugewinn an Macht und dem eigenen Machtverlust aufkommen, beispielsweise beim Wunsch der Bewahrung der Grenze in Bezug auf das, was Homosexuelle dürfen sollen und was nicht. Dafür könnte auch sprechen, dass Homosexuelle zwar akzeptiert werden, jedoch nur, wenn diese die als eigen empfundenen Bereiche, wie z. B. den der Ehe, nicht berühren. Das legt die Überlegung nahe, dass der vermutete Angriff vielmehr einer gefürchteten Auflösung der Grenze zwischen Homo- und Heterosexualität geschuldet ist, wenn Elternschaft nicht zwangsläufig heterosexuell gedacht wird. Darauf könnte auch die massive Kritik an den Gendertheorien und Butler verweisen, deren Theorien für diese «entgrenzende» Entwicklung verantwortlich gemacht werden. An der 
Stelle, an der Homosexuellen das Recht auf Ehe zugesprochen werden könnte, zeigt sich ein Bedürfnis nach dem Vorhandensein einer Differenz, die bestehen bleiben müsste, um gemäss des Narzissmus Alterität und damit Sexualität zu vermeiden und sich von denjenigen, bei denen das Sexuelle verortet wird, und folglich im doppelten Sinne vom Sexuellen abzugrenzen.

Für einen Verteilungskampf könnte auch der Neid auf homosexuelles Begehren sprechen. Herbert Gschwind vermutet beispielsweise in Charles Socarides Eifer, homosexuelle Männer zu heilen, einen potenziell neidgeprägten Wunsch, diese Männer und ihr Begehren seinem eigenen anzugleichen, um deren «Lust auf sein heterosexuelles Maß zu bringen» (2015, S. 644). Die Homophobie wäre in dieser Logik darin begründet, dass ein potenzielles Mehr auf Homosexuelle projiziert und beneidet würde (ebd.). Davon ausgehend wäre zu fragen, ob es sich bei der vielen Kritik an der Grenzenlosigkeit in den unterstellten Wünschen nach Allmacht auch um Neid auf die Möglichkeit, mehr haben zu können, handeln könnte. So wird dem Anderen ein Mehr unterstellt, etwa die Möglichkeit, genussvolle Erschütterung oder Selbstauflösung zu erfahren - der Neid bezöge sich dann auf die Möglichkeit, die Entgrenzung und damit das Sexuelle zu erfahren.

In den dargestellten Positionen scheint zudem bereits die Tatsache, mehr haben zu wollen, für Erregung zu sorgen. Während diese Mehrheit die Bedeutung von Intimität und klaren Grenzen im Sinne der Tugend des Verzichts an Möglichkeiten (der Ehe für alle, der Begrenztheit der Geschlechtlichkeit) proklamiert, wird die Forderung nach einem Mehr den Anderen unterstellt (oder an die Anderen delegiert) und abgelehnt. Die Ehe könnte in diesem Zusammenhang als ein Privileg gesehen werden, welches die Heterosexuellen beanspruchten, und was im Gegensatz zum Privileg des Ausgeliefertseins oder der Möglichkeit zu begehren stünde (s. o.). Damit könnte das Bedürfnis nach Abgrenzung und Differenz auch das Resultat eines Verteilungskampfes sein. Es handelt sich demnach sowohl um eine Abwehr des Sexuellen im Sinne einer Abgrenzung zur Vermeidung der Überschreitung oder der Verführung als auch um die von Pfaller beschriebene Ablehnung von Alterität im Sinne des Narzissmus, was wiederum zur Ablehnung des Sexuellen führt. Zudem könnte ein Aushandlungskampf im Sinne des Begehrens von Bedeutung sein, der sich um die Frage dreht, wer derzeit (a)sexuell ist.

Wenn sich in der männlichen Homosexualität und in der wiedergegebenen Diskussion um unter anderem die Homo-Ehe das bedrohliche wie beneidete Sexuelle zeigt, könnte die ablehnende oder homophobe Haltung als der Moment betrachtet werden, in welchem sich das Aufscheinen des Sexuellen mit dessen 
Vertreibung verbindet. Die Homophobie wäre damit im Sinne der Abwehr als eine Lösung des Widerspruchs zu sehen, das Sexuelle sowohl abzulehnen oder den Verzicht libidinös zu besetzen und gleichzeitig über die Ablehnung der Anderen daran teilzuhaben. Über das Aufrechterhalten des Anderen-Status - der Differenz könnte das Sexuelle erhalten und als «exotisch» (Pfaller, 2009, S. 41) bestaunt und auf Distanz gehalten werden.

\section{Sexuelle Vielfalt und Psychoanalyse}

Auch wenn Homosexuelle in den Ausbildungsinstituten nach wie vor wenig präsent sind, scheint sich deren Situation in den Instituten teilweise verbessert zu haben (Rauchfleisch, 2014). Der Eröffnungsvortrag an der Jahrestagung der Deutschen Gesellschaft für Analytische Psychologie (DGAP) thematisierte und kritisierte die anhaltende Homophobie in der Psychoanalyse (Herzog, 2015), die Konferenz der International Psychoanalytical Association (IPA) 2015 befasste sich mit "new family configurations; sexual and gender identity; race and racism; homophobia, transphobia and biphobia" (IPA, 2015). Die Deutsche Psychoanalytische Vereinigung (DPV) lud zu ihren Jahrestagungen Gäste wie Christina von Braun und Gschwind ein, ${ }^{9}$ die unter anderem die Homophobie sowie die jahrelange Diskriminierung von Homosexuellen durch die Psychoanalyse feststellen und kritisieren. Einige Institute setzten sich im letzten Jahr weiter mit Homophobie oder der Sinnhaftigkeit der Unterscheidung von Hetero- und Homosexualität auseinander. ${ }^{10}$ Auch die Zeitschriften «Psyche 07/15», die «Freie Assoziation 1/2016» sowie dieses Journal widmen Fragen zu Sexualität, sexuellen Verhältnissen oder Homo- und Heterosexualität eine Ausgabe. Nicht nur aus der Perspektive der von Diskriminierung und Ausschluss aus der Psychoanalyse Betroffenen ist es zu begrüssen, dass diese Auseinandersetzungen stattfinden. Gleichwohl ist es aus einer psychoanalytischen Perspektive bedeutsam, auch diese Entwicklung einer Reflexion hinsichtlich ihrer verschiedenen Ursachen und Motive zu unterziehen. Wie ist nun unter der Annahme der Wirkung unbewusster Wünsche und Ängste bei der Bewertung der Homosexualität das Interesse an einer Auseinandersetzung mit Homosexualität und «sexuellen Verhältnissen» zu verstehen? Gibt es Zusammenhänge zwischen dieser Entwicklung und dem aktuellen Stand der Psychoanalyse (u. a. Kandidat_innenschwund in den psychoanalytischen Ausbildungsinstituten, die bereits erwähnte «Entsexualisierung» psychoanalytischer Theorie)? Finden sich weiter Parallelen zu den homophoben Mechanismen? Die folgenden Überlegungen sollen eher Fragen und Diskussionen anstossen, als eine Erklärung oder Analyse der Situation bieten. 
Die Bewertung der Homosexualität als pathologisch und der Ausschluss homosexueller Kandidat_innen von der psychoanalytischen Ausbildung wurden vielfach als Anpassung an die gesellschaftlichen Verhältnisse diskutiert. So wurden die durch den Nationalsozialismus bedingte Emigration in die USA und die auf die Sicherung der beruflichen und finanziellen Situation zielende Anpassung an die homophobe McCarthy-Politik genannt (Pechriggl, 2012, S. 96). ${ }^{11}$ Von der Annahme einer Anpassung an homophobe Verhältnisse ausgehend, wäre zu überlegen, inwiefern ein offener Umgang als eine Anpassung an den gesellschaftlichen Wandel zu bewerten wäre, in welcher Vielfalt ein Ideal darstellt (s. o.). Inwiefern wird homosexuelles Begehren seitens der Gesellschaft nach wie vor als eine Bedrohungwahrgenommen - oder ist es nicht vielmehr der anhaltende Vorwurf der Homophobie, der als bedrohlich für die Psychoanalyse zu bewerten ist? Welches Bild will man von sich vermitteln, und verschiebt sich eventuell das Ideal des Normindividuums von einem heterosexuellen zu einem queeren? ${ }^{12}$ Gerade weil es aktuell nicht sehr gut um den Ruf der klinischen Psychoanalyse und ihren Nachwuchs zu stehen scheint, könnte es sich bei der konstatierten Öffnung auch um einen Versuch handeln, zeitgemäss zu wirken, was wiederum auf eine Abwehrbewegung hinweisen könnte. Weiter könnte es einen Versuch darstellen, dem Nachwuchsmangel entgegen zu steuern. Denn

[d]er Toleranz- und Antidiskriminierungsdiskurs verändert das Verhalten gegenüber sexuellen Minderheiten insofern, als sie gesellschaftlich integrierbar und damit ökonomisch verwertbar und beschäftigungsfähig gemacht werden können. (Mayrhofer, 2012, S. 73)

Auch sollte die Art und Weise, wie die Auseinandersetzungen über sexuelle Verhältnisse geführt werden, einer Betrachtung unterzogen werden. Denn in den von mir besuchten Veranstaltungen und anschliessenden Diskussionen zu diesen Themen blieben Kontroversen in der Regel aus, und fast im Konsens wurden homophobe Praktiken kritisiert. Ist das Homophobe damit lediglich ausserhalb dieser Auseinandersetzungen zu verorten oder gibt es auch in der offenen Auseinandersetzung, wie Barbara Zach vermutet, Hemmungen, darüber zu sprechen?

Der freien Rede ging unter der drückenden Last des psychoanalytischen Erbes die Luft aus. Freiberg war auch kein Monte Veritá, 
der zur Erprobung neuen psychoanalytischen Denkens eingeladen hätte. Oder vielleicht doch, es gab die Einladung, doch niemand folgte ihr, obwohl viele gekommen waren. (Zach, 2014)

Sofern also etwas ausgespart wird: Was ist es, das nicht zu Wort kommt oder abgewehrt wird? Sind es eigene homophobe Anteile oder ist es vielmehr die Angst, als solche Person deklariert zu werden? Oder sind es Schuldgefühle, sich nicht gegen das damals wie heute bestehende Unrecht eingesetzt und das Privileg des Zugangs zur Ausbildung besessen zu haben?

Mit Bezug auf Kunstreich, der einen gesellschaftlichen Trend zu queer kritisiert, weil dieser den Blick auf die Homophobie versperre, wäre zu überlegen, inwiefern ein Fokus auf sexuelle Vielfalt dann wiederum einem Aussparen der Schuld gegenüber Homosexuellen gleichkäme (Kunstreich, 2015, S. 73).

Welche verschiedenen Motivationen können der Beschäftigung mit beispielsweise der Homophobie in der Psychoanalyse zugrunde liegen? Welche Vorstellung von der Psychoanalyse besteht dabei? Gibt es ein Bild einer idealen Psychoanalyse, welche, dieser Vorstellung nach, lediglich falsch angewendet wird? Würde sich darin auch ein Wunsch nach einer ursprünglichen und unmittelbaren Psychoanalyse Freuds und seiner Haltung gegenüber Homosexuellen und homosexuellem Begehren zeigen? Diese wird vielfach als ambivalent, aber dennoch offen beschrieben (u. a. Gschwind, 2015, S. 645f.) und wiederholt als Begründung herangezogen, die Psychoanalyse habe das Potenzial, die Mechanismen der Homophobie zu begreifen. Damit wäre eine homosexuellenfreundliche Psychoanalyse auch ein Sinnbild für die einst offene, dynamische, noch unschuldige Psychoanalyse.

Folglich wäre eine deklarierte Offenheit auf deren Gehalt zu prüfen und zu fragen, inwiefern diese dem Bedürfnis, den Nachwuchsmangel in den Griff zu bekommen, oder einem Wunsch nach Wiedergutmachung geschuldet ist. Würden sich die Grenzen der Offenheit lediglich verschieben? Inwiefern handelt es sich um eine Offenheit gegenüber einer «monogamen, eheähnlichen Homosexualität», und wie werden aber z. B. Bisexualität oder Promiskuität bewertet? Handelt es sich bei einer potenziellen Toleranz nicht eher um eine Folklorisierung des Anderen im Sinne Slavoj Žižeks? So würde die «Verkleidung» der Anderen akzeptiert und die Anerkennung gegeben, solange sich der Andere den Werten und Forderungen der Mehrheit unterwirft (Hetzel, 2006, S.242). Von der vorherigen Analyse ausgehend, ist zudem die Frage bedeutsam, inwiefern Toleranz nur dann nicht an ihre Grenzen kommt, solange das Mehr des Anderen nicht zu bedrohlich ist. 


\section{Literatur}

Bersani, L. (1988). Ist das Rectum ein Grab? Lettre International (3), 52-58.

Braun v., Ch. (2014). Blutslinien - Wahlverwandtschaften. Genealogien aus psychoanalytischer und kulturhistorischer Sicht. Psyche 68 (8), 393-418.

Dannecker, M. (2014). Von den Schwierigkeiten der Psychoanalyse mit der männlichen Homosexualität. texte. psychoanalyse. ästhetik. kulturkritik 34 (2), 34-82.

Eder, F. (2010). Liberalisierung und Kommerzialisierung der Sexualität in der zweiten Hälfte des 20. Jahrhundert. In Th. Benkel \& F. Akalin (Hrsg.), Soziale Dimensionen der Sexualität (S. 153-176). Giessen: Psychosozial.

Freud, S. (1905d). Drei Abhandlungen zur Sexualtheorie. GWV, 29-159.

Freud, S. (1937c). Die endliche und die unendliche Analyse. GWXVI, 59-99.

Green, A. (1995). Has sexuality anything to do with psychoanalysis? International Journal for Psychoanalysis 76 (5), 871-83.

Gschwind, H. (2015). «Manif[est] Homos[exuelle] wären - einstweilen - grundsätzlich abzuweisen. Sie sind ja meist zu abnorm». Zum Verhältnis von Psychoanalyse und Homosexualität. Psyche 69 (7), 632-647.

Härtel, I. (2014). Ego-dissolution? Homosexualität als Denkfigur - kulturtheoretische Betrachtungen. texte. psychoanalyse. ästhetik. kulturkritik 34 (2), 99-118.

Hetzel, A. \& Hetzel, M. (2006). Slavoj Žižek: Psychoanalyse, Idealismus und Populärkultur. In S. Moebius \& D. Quadlieg (Hrsg.), Kultur. Theorien der Gegenwart (S. 235-245). Wiesbaden: VS Verlag für Sozialwissenschaften.

Heenen-Wolff, S. (2009). Abschied vom Schiboleth? Über das Verschwinden der Sexualität in der zeitgenössischen Psychoanalyse. In I. Berkel (Hrsg.), Postsexualität. Zur Transformation des Begehrens (S. 169-190). Giessen: Psychosozial.

Herzog, D. (2015). Die bemerkenswerte Beständigkeit der Homophobie in der Psychoanalyse. Öffentlicher Vortrag zur Eröffnung der DGAP-Tagung 2015 EROS. Liebe-Sex-Perversion in Berlin.

IPA (International Psychoanalytic Association) (2015). Programm zur Tagung, http:// www.ipa.org.uk/Congress/Congress_Programme/Congress_ Themes/Programme.aspx (25.12.15).

Keller, G. 22. 1.2015. Homophobie beim Psychologen. Die Behandlung. tageszeitung, http://www.taz.de/!5023685/ (26.01.16).

Kunstreich, T. (2015). Dialektik der Abweichung. Über das Unbehagen in der homosexuellen Emanzipation. Hamburg: KVV konkret. 
Laplanche, J. (1996). Die unvollendete kopernikanische Revolution. Frankfurt a. M.: Fischer.

Mayrhofer, M. (2012). Queeuropa. Toleranz und Antidiskriminierung von LGBT als Technologie der neoliberalen Gouvernementalität der europäischen Integration. In H. Haberler et. al. (Hrsg.), Queer zum Staat. Heteronormativitätskritische Perspektiven auf Staat, Macht und Gesellschaft (S. 61-77). Berlin: Querverlag.

Metzger, H.-G. (2015a). Konflikte zwischen Psychoanalyse und Gendertheorie Väter und die neuen Formen der Elternschaft. Psychoanalyse Aktuell, http:// www.psychoanalyse-aktuell.de/artikel/detail/news/dr-hans-geert-metzger-konflikte-zwischen-psychoanalyse-und-gendertheorien-vaeter-unddie-neuen-formen-der-elternschaft/ (26.01.16).

Metzger, H.-G. (2015b). Conchita Wurst und die Illusionen in den Gendertheorien. Psychoanalyse Aktuell, http://www.psychoanalyse-aktuell.de/artikel/detail/ news/hans-geert-metzger-conchita-wurst-und-die-illusionen-in-den-gendertheorien/? (26.01.16).

Pechriggl, A. (2012). Homophobie und die Dialektik der Selbstaufklärung in der Psychoanalyse. In M. Bidwell-Steiner \& A. Babka (Hrsg.), Obskure Differenzen. Psychoanalyse und Gender Studies (S. 83-99). Giessen: Psychosozial.

Pfaller, R. (2005). Der Normalnarzissmus der Verhandlungsmoral und seine Widersacherin, die Normalperversion - und was die Psychoanalyse aus ihrem Gegensatz lernen kann. Zu Franz Oberlehners Text «Sexualität und Bindung im Spätkapitalismus. Von der Normalneurose zur Normalperversion». texte. psychoanalyse. ästhetik. kulturkritik 25 (4), 7-21.

Pfaller, R. (2009). Strategien des Beuteverzichts. Die narzisstischen Grundlagen aktueller Sexualunlust und Politohnmacht. In I. Berkel (Hrsg.), Postsexualität. Zur Transformation des Begehrens (S. 31-48). Giessen: Psychosozial.

Psyche (2015). Sexuelle Verhältnisse 69 (7).

Rauschfleisch, U. (2014). Homosexualität und Psychologie/ Psychoanalyse. In F. Mildenberger et. al. (Hrsg.), Was ist Homosexualität? Forschungsgeschichte, gesellschaftliche Entwicklungen und Perspektiven (S. 357-389). Hamburg: Männerschwarm.

Schmidt, G. (1996). Das Verschwinden der Sexualmoral. Über sexuelle Verhältnisse. Hamburg: Klein.

Shalev, O. \& Yerushalmi, H. (2009). Status of sexuality in contemporary psychoanalytic psychotherapy as reported by therapists. Psychoanalytic Psychology 26, 343-361. 
Sigusch, V. (2013). Sexualitäten. Eine kritische Theorie in 99 Fragmenten. New York, Frankfurt a. M.: Campus.

Quindeau, I. (2015). «Recovering from Iatrogenesis ...» Vom Umgang mit dem homophoben Erbe. Psyche 69 (7), 648-660.

Vacquin, M. \& Winter, J.-P. (2013). Schluss mit Mutter und Vater, http://www.freiewelt.net/blog/schluss-mit-vater-und-mutter-5476/ (15.12.15). Original: (2013). Pour en finir avec père et mère. Le Débat 174, S. 84-89.

Zamanian, K. (2011). What Happened to Infantile Sexuality? Psychoanalytic Psychology 28, 33-47.

Zach, B. 04.09.2014. Freiberg 2014 oder die Hemmung über Homosexualität zu sprechen, in Queering Psychoanalysis, https://queeringpsychoanalysis. wordpress.com/2014/09/04/freiberg-2014-oder-die-hemmung-uberhomosexualitat-zu-sprechen/ (26.01.16).

\section{Anmerkungen}

1 Wenn ich im Folgenden von Homosexualität spreche, meine ich nicht, dass es die eine Homosexualität an sich gibt. Vielmehr geht es mir dabei um das kulturell vorherrschende Bild von Homosexualität.

2 Ich verwende die Begriffe «Trieb», «Sexuelles» und «Sexualität» in der folgenden Arbeit weitgehend synonym, obwohl dies terminologisch ungenau ist.

3 Selbstverständlich gibt es die Psychoanalyse nicht und so kann diese nicht als einheitliches, in sich geschlossenes Gebilde gedacht werden. Sie ist potenziell insofern einem Wandel unterzogen, als dass die jeweiligen Mitglieder oder psychoanalytisch Denkenden eben die Psychoanalyse ausmachen. Da sie jedoch u. a. auch in Form der Ausbildungsstätten und Verbände institutionalisiert ist, spreche ich in diesem Artikel von der Psychoanalyse.

$4 \quad$ Dannecker geht mit Freud von einer bisexuellen Anlage des Menschen aus und verweist auf das Konzept der latenten Homosexualität - wobei er von der Tragfähigkeit dieses Konzeptes nicht voll überzeugt ist (2014, S. 78). Angenommen wird dabei, dass latente Homosexualität Konflikte generiert, sobald sie aufgrund individueller Ängste oder gesellschaftlicher Normvorstellungen unterdrückt werde. Dannecker verweist weiter auf Freud, der den heterosexuellen Mann in einem anhaltenden Konflikt mit seiner Bisexualität sah (Freud, 1937, S. 89, zit.n. Dannecker ebd.), sowie darauf, dass «homosexuelle Männer in [der Vorstellungswelt der heterosexuellen Psychoanalytiker] eine Gefahr nicht nur für ihre männliche Identität, sondern auch für ihre Werte von Männlichkeit repräsentieren» (ebd.).

$5 \quad$ Der Text von Vacquin und Winter wurde von Andreas Lombard auf dem Blog Die freie Welt. Die Internet- \& Blogzeitung für die Zivilgesellschaft veröffentlicht, der laut Lobbypedia der Alternative für Deutschland (AFD) nahesteht (https://lobbypedia.de/wiki/FreieWelt.net [30.01.16]).

6 Dagegen kann eingewendet werden: Natürlich kann in einer homosexuellen Elternschaft nach der Bedeutung des nicht präsenten Gegengeschlechts und nach potenziellen Ausschlüssen gefragt werden. Ebenso wie in einer heterosexuellen Beziehung die potenziell ausgeschlossenen homosexuellen Anteile auf deren Bedeutung geprüft werden können.

$7 \quad$ Auch an anderen Stellen oder in anderen Figuren kann Abgewehrtes und das Sexuelle aufscheinen (Härtel, 2014).

8 Für diesen und weitere Hinweise danke ich Sonja Witte. 
9 Der, laut eigener Angabe, selbst überrascht über die Einladung zu sein schien (Gschwind, 2015, S. 632).

10 So z. B. Quindeau in Berlin http://www.apb.de/veranstaltungen/ [30.01.16] oder Rudolf in Bamberg http://www.psychoanalyse-bamberg.de/index.php/veranstaltungen/g-6-_c8sm2chk6gojcbb360sm2b9k6ss30bb1ccsm6bb36sp34d9gcks3aohp74_201410241700 [30.01.16] oder in Hamburg http://www.mbi-hh.de/PDF/wise_2015-2016.pdf [30.01.16].

11 Anna Freud lehnte zeitweise die Aufnahme homosexueller Ausbildungskandidat innen strikt ab, weil sie um den Ruf der Psychoanalyse besorgt war (Pechriggl, 2012, S. 96).

12 So sieht Pechriggl mit Bezug auf Bions Grundannahmen der Paarung in den psychoanalytischen Ausbildungsinstituten ein unbewusstes kollektives Ideal eines heterosexuellen Normindividuums, welches für das Fortleben und damit die Existenz der Psychoanalyse von Bedeutung sei. In dieser Logik stellten homosexuelle Ausbildungskandidat_innen diesen phantasierten Idealtypus der Analytiker_innen in Frage, weshalb diese abgelehnt würden (Pechriggl, 2012).

\section{Angaben zur Autorin}

Victoria Preis, 1985, ist Kulturwissenschaftlerin (B. A.) und studiert Psychologie (M.A.) an der IPU Berlin. Ihre Schwerpunkte sind Fragen an der Schnittstelle zwischen Psychoanalyse und Geschlechterfragen. 